\title{
Chinesische Beteiligungen und Übernahmen in Deutschland
}

In den Jahren 2017 und 2018 verschärfte die Bundesregierung jeweils die Außenwirtschaftsverordnung, um sich bei Übernahmen und Beteiligungen ausländischer Investoren im Inland mehr Befugnisse zu sichern. Auch 2020 ist eine weitere Verschärfung geplant. ${ }^{1}$ Zusätzlich wurde im Jahr 2019 - insbesondere auf Initiative Deutschlands $^{2}$ - in der EU-Verordnung 2019/452 die Schaffung eines Rahmens für ausländische Direktinvestitionen auf EU-Ebene beschlossen. Hintergrund dieser Eingriffe ist der wirtschaftliche Aufstieg der Volksrepublik China und die damit zusammenhängende zunehmende Aktivität chinesischer Investoren in Deutschland und auch weltweit. Befürchtet wird unter anderem, dass mit Hilfe staatlich gelenkter Investitionsprogramme wie „Made in China 2025“ der wirtschaftliche Aufstieg Chinas in unfairer Weise vorangetrieben wird. ${ }^{3}$ Ziel der ergriffenen Maßnahmen soll somit lediglich sein, durch China ausgelöste Wettbewerbsverzerrungen zu beheben. Dieser Beitrag hat sich vor diesem Hintergrund zum Ziel gesetzt, die chinesischen Beteiligungen und Übernahmen in Deutschland quantitativ zu erforschen, um eine realistische Einschätzung der Aktivitäten chinesischer Investoren zu ermöglichen.

(C) Der/die Autor(en) 2020. Open Access: Dieser Artikel wird unter der Creative Commons Namensnennung 4.0 International Lizenz (https:// creativecommons.org/licenses/by/4.0/deed.de) veröffentlicht.

Open Access wird durch die ZBW - Leibniz-Informationszentrum Wirtschaft gefördert.

1 Vgl. Bundesministerium für Wirtschaft und Energie: Industriestrategie 2030, November 2019, S. 27.

2 Vgl. Bundesministerium für Wirtschaft und Energie: Stärkung unserer nationalen Sicherheit durch verbesserte Investitionsprüfung, Pressemitteilung vom 19.12.2018, https://www.bmwi.de/Redaktion/ DE/Pressemitteilungen/2018/20181219-staerkung-unserer-nationalen-sicherheit-durch-verbesserte-investitionspruefung.html (22.1.2020).

3 Vgl. S. Beer, J. Matthes, C. Rusche: Decoupling Chimerica, Consequences for the European Union, IW-Report, Nr. 42, Köln 2019.

\section{Chinesische Direktinvestitionen}

Dazu wird zunächst die Entwicklung von ausländischen Direktinvestitionen in die und aus der Volksrepublik China analysiert. Die Betrachtung von Direktinvestitionen ermöglicht es, langfristige Investitionen mit strategischem Einfluss auf das Investitionsobjekt in den Fokus zu nehmen, da nur Transaktionen mit mehr als $10 \%$ der Stimmrechte betrachtet werden. ${ }^{4}$ Darunter fallen Übernahmen von und Beteiligungen bei Unternehmen sowie Unternehmensneugründungen. In Bezug auf die Volksrepublik China (vgl. Abbildung 1) kann festgehalten werden, dass das Land zu Beginn der Öffnungspolitik 1979 weder als Ziel noch als Ursprung von Direktinvestitionen eine Rolle gespielt hat. Erst in den 1990er Jahren wurde China als Ziel von Investitionen zunehmend attraktiv. Am Ende der 1990er Jahre wurden so mehr als 40 Mrd. US-\$ im Jahr investiert. Mit Ausnahme von leichten Rücksetzern um das Jahr 2000 und in der Finanzkrise stiegen die Investitionen danach in China kontinuierlich auf rund 139 Mrd. US-\$ im Jahr 2018, dem letzten Jahr, für das bereits Zahlen vorliegen. Auch wenn der Anstieg an Investitionen in den vergangenen Jahren abgeflacht ist, ist die Volksrepublik dennoch ein sehr attraktives Ziel für ausländische Direktinvestitionen.

Als Ursprung von Direktinvestitionen erlangte die Volksrepublik erst mit Beginn dieses Jahrtausends Bedeutung. Neben dem Beitritt zur Welthandelsorganisation (WTO) 2001 dürfte insbesondere die Going-Out-Strategie der chinesischen Regierung von $2002^{5}$ eine Rolle gespielt haben. So stiegen die Investitionen aus China von lediglich 3 Mrd. US-\$ im Jahr 2003 auf mehr als 196 Mrd. US-\$ im Jahr 2016. Danach fielen die Investitionen auf nahezu 130 Mrd. US-\$ im Jahr 2018. Der Rückgang in den Jahren 2017 und 2018 ist dabei wohl zum einen auf die Verschärfung der chinesischen Regelungen im
Dr. Christian Rusche ist Economist für Industrieökonomik und Wettbewerb beim Institut der deutschen Wirtschaft Köln.
4 Vgl. Organisation for Economic Co-operation and Devlopment (OECD): OECD Benchmark Definition of Foreign Direct Investment, 4. Aufl., Paris 2008, S. 17.

5 Vgl. G. Lim: China's "Going Out“ Strategy in Southeast Asia: Case Studies of the Automobile and Electronics Sectors, in: China: An International Journal, 15. Jg. (2017), H. 4. S. 157-178. 


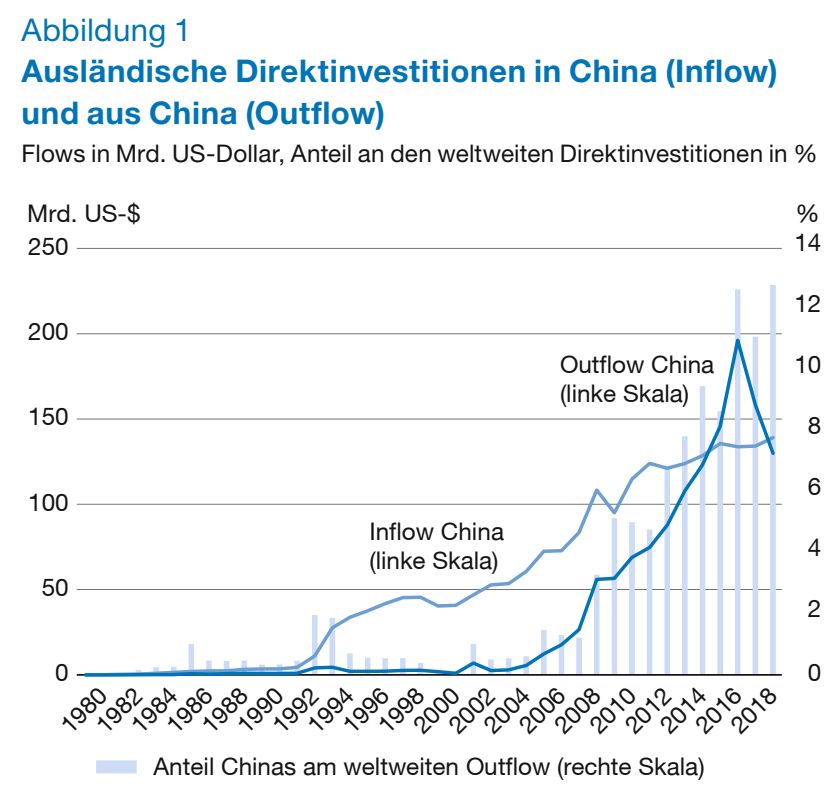

Quelle: UNCTAD Data Center: Foreign direct investment: Inward and outward flows and stock, annual, https://unctadstat.unctad.org/wds/TableViewer/tableView.aspx?Reportld=96740 (21.1.2020).

Jahr 2016 zurückzuführen. ${ }^{6}$ Durch die verstärkte Kontrolle sollten insbesondere unproduktive Investitionen vermieden werden. Zum anderen dürften auch die verschärften Regeln unter anderem in Deutschland sowie der Handelskonflikt mit den USA ${ }^{7}$ eine Rolle gespielt haben. Trotz des Rückgangs in absoluten Werten hat der Anteil der Direktinvestitionen aus China an den weltweit getätigten ausländischen Investitionen mit nahezu $13 \%$ (2018) sogar ein neues Allzeithoch erreicht.

Es bleibt festzuhalten, dass Investitionen aus China weltweit gesehen immer mehr an Bedeutung gewonnen haben. Zudem ist die Volksrepublik in den Jahren 2015 bis 2017 erstmals zu einem Nettoinvestor geworden, d. h. die Direktinvestitionen aus China überstiegen die Investitionen in China. Mit dem Erreichen einer ersten Einigung im Handelsstreit mit den USA sowie vor dem Hintergrund zunehmender chinesischer Investitionen im Rahmen der Belt-and-Road-Initiative ${ }^{8}$ sollte China als Investor auch nach 2018 weiter an Bedeutung gewinnen.

6 Vgl. C. Rusche: Chinesische Investoren in Deutschland in 2017 sehr aktiv, IW-Kurzbericht, Nr. 11, Köln 2018

7 Vgl. S. Beer, J. Matthes, C. Rusche, a. a. O.

8 Ebenda.

\section{Abbildung 2}

\section{Chinesische ${ }^{1}$ Übernahmen und Beteiligungen in}

\section{Deutschland}

Zahl insgesamt, Zahl mit veröffentlichten Transaktionswerten sowie Summen der veröffentlichten Werte in Mrd. Euro

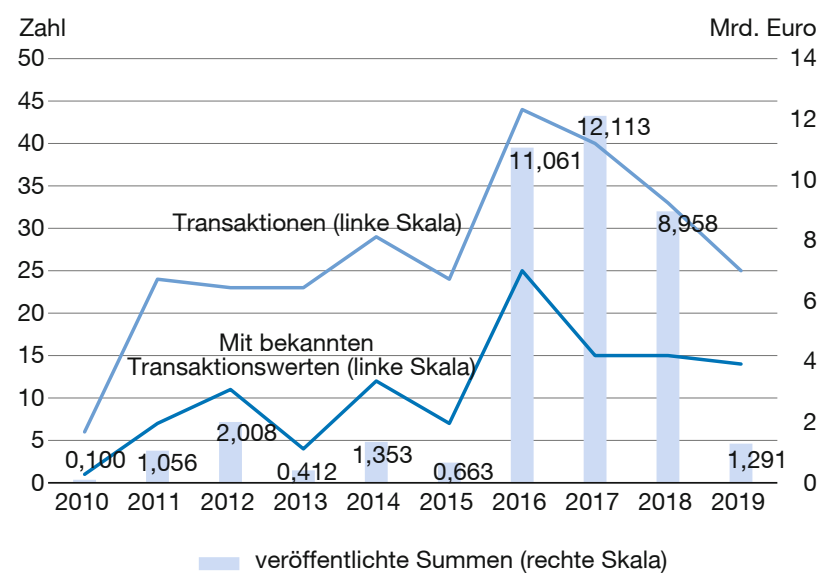

${ }^{1}$ Volksrepublik China und Hongkong.

Quelle: eigene Recherchen.

\section{Chinesische Übernahmen und Beteiligungen in Deutschland}

Die Direktinvestitionen Chinas sind dabei jedoch sehr ungleich verteilt. Im Jahr 2017 flossen mehr als $57 \%$ aller chinesischen Investitionen nach Hongkong. ${ }^{9} 11,7 \%$ entfielen auf Europa (Kontinent) und 4,1 \% auf die USA. Diese Ungleichheit bei den Flüssen an Direktinvestitionen spiegelt sich auch bei den Beständen an Direktinvestitionen wider. Auf Hongkong entfielen 2017 mehr als $54 \% .^{10}$ Auf ganz Europa entfielen lediglich 6,1\% und auf die USA 3,7\%. Somit ist auch die Bedeutung Deutschlands als Ziel chinesischer Direktinvestitionen eher gering: Der Anteil Deutschlands an chinesischen Direktinvestitionen lag bisher im niedrigen einstelligen Prozentbereich. ${ }^{11}$

Dementsprechend sind auch die chinesischen Übernahmen und Beteiligungen in Deutschland vergleichsweise gering (vgl. Abbildung 2). Aufgrund der engen Verflechtung zwischen Hongkong und der Volksrepublik, werden dabei beide zusammen betrachtet. Bis einschließlich 2010 spielten chinesische Übernahmen keine Rolle und betrafen nur sehr wenige Fälle. ${ }^{12}$ Beispiels-

9 Ebenda, S. 23.

10 Ebenda, S. 31.

11 C. Rusche: Aktivitäten chinesischer Investoren in Deutschland, in: IWTrends, 44. Jg. (2017), H. 2, S. 41-59, S. 51.

12 Vgl. C. Rusche: Aktivitäten chinesischer Investoren ..., a. a. O. 
weise konnten 2010 lediglich sechs Transaktionen verzeichnet werden. Auch bei diesen Angaben werden nur Transaktionen betrachtet, bei denen mindestens $10 \%$ der Stimmrechte übernommen werden. Davon wird lediglich abgewichen, wenn es sich um ein bedeutendes Unternehmen handelt und der Anteil nahe an den $10 \%$ liegt. Zu nennen wären hier beispielsweise der Einstieg des chinesischen Autobauers Geely bei der Daimler AG mit 9,7\% im Jahr 2018 oder die zwischenzeitliche Beteiligung von 9,9\% der HNA-Group an der Deutschen Bank AG im Jahr 2017.

Eine Zunahme der Fallzahlen trat erst ab 2011 ein. In diesem Jahr konnten 24 Transaktionen verzeichnet werden. Mit Ausnahme des Jahres 2014, in dem 29 Transaktionen gezählt wurden, konnten bis einschließlich 2015 jährlich lediglich 24 bzw. 23 Transaktionen verzeichnet werden. Im Jahr 2016 verdoppelte sich die Zahl im Vergleich zum Vorjahr jedoch nahezu auf 44 Fälle. Seit diesem Höhepunkt gehen die Fallzahlen analog zur generellen Entwicklung chinesischer Direktinvestitionen weltweit kontinuierlich zurück. So konnten im vergangenen Jahr erneut lediglich 25 Transaktionen verbucht werden. Zu diesem Rückgang haben auch die Tätigkeiten der Bundesregierung beigetragen: Die staatliche Kreditanstalt für Wiederaufbau musste sich 2018 beim Netzbetreiber 50 Hertz beteiligen, um einem chinesischen Staatsunternehmen zuvorzukommen. ${ }^{13}$ Zudem kamen die beschlossenen Verschärfungen der Außenwirtschaftsordnung zur Anwendung, wodurch 2018 ebenfalls eine weitere Transaktion verhindert wurde. ${ }^{14}$ Auch 2019 kamen die erweiterten Kompetenzen insbesondere beim Verkauf des Geschäftsfeldes Locomotives der Vossloh AG an eine Tochtergesellschaft der China Railway Rolling Stock Corporation (CRRC) ${ }^{15}$ zur Anwendung. Die Prüfung dieser Transaktion dauert bis heute (24.1.2020) an. Befürchtet wird, dass durch diese vergleichsweise kleine Übernahme mit einem Wert im einstelligen Millionenbereich dem chinesischen Unternehmen der Einstieg in den europäischen Markt geebnet wird..$^{16}$

Durch den Rückgang der Fallzahlen nahm ebenfalls die relative Bedeutung chinesischer Investoren bei den Übernahmen und Beteiligungen in Deutschland ab.

13 C. Rusche: Chinesische Beteiligungen und Übernahmen 2018 in Deutschland, IW-Kurzbericht, Nr. 5, Köln 2019.

14 Ebenda.

15 Vossloh AG: Vossloh Aktiengesellschaft: Vossloh unterzeichnet Vertrag zum Verkauf des Geschäftsfelds Locomotives, 26.8.2019, https://www.dgap.de/link.php?isin=DE0007667107\&typ=adhoc\&von=1 9970101\&a $=10 \&$ sprache $=$ de\&v $=$ de\&id $=1188519$ (23.1.2020).

16 Ntv: China kauf Vossloh-Lok-Spartte: Le Maire macht Brüssel schwere Vorwürfe, 1.9.2019, https://www.n-tv.de/wirtschaft/Le-Mairemacht-Bruessel-schwere-Vorwuerfe-article21244116.html (24.1.2020).
Bezogen auf die Transaktionen entfielen 2017 lediglich $6,6 \%{ }^{17}$ auf Investoren aus der Volksrepublik China und Hongkong. 2018 waren es geschätzt nur noch $5,5 \%{ }^{18}$, und auf Basis der Zahlen der PricewaterhouseCoopers Wirtschaftsprüfungsgesellschaft sank der Anteil 2019 sogar auf $5,3 \% .^{19}$

Der Wert der chinesischen Transaktionen in Deutschland ist in den vergangenen Jahren mit den Fallzahlen ebenfalls rapide gesunken. 2019 entsprachen die Transaktionen lediglich einem Wert von rund 1,3 Mrd. Euro und damit ungefähr dem Niveau der Jahre 2011 bis 2015. Die höchsten Transaktionswerte wurden 2016 mit mehr als 11 Mrd. Euro und 2017 mit mehr als 12,1 Mrd. verzeichnet. Diese hohen Werte waren jedoch auf große Einzeltransaktionen zurückzuführen. ${ }^{20}$ Auch der hohe Wert im Jahr 2018 ist durch eine Einzeltransaktion geprägt: Der Einstieg bei der Daimler AG trug mehr als $7 \mathrm{Mrd}$. Euro bei. Bei der Betrachtung der Transaktionswerte muss jedoch beachtet werden, dass nicht alle Werte veröffentlicht werden.

\section{Fazit zu chinesischen Beteiligungen und Übernahmen in Deutschland}

Seit dem Höhepunkt chinesischer Investitionen im Ausland 2016 sind die Aktivitäten weltweit, aber auch speziell in Deutschland merklich zurückgegangen. Dazu beigetragen haben regulatorische Verschärfungen in und außerhalb Chinas, aber auch Unsicherheiten im Zuge der aktuellen Handelsstreitigkeiten. Folglich könnten bei einer Verminderung der Unsicherheiten und generell durch eine wachsende Bedeutung der Volksrepublik in der Weltwirtschaft sowie die zunehmende Handelsverflechtung über die Belt-and-RoadInitiative auch die Aktivitäten chinesischer Investoren wieder zunehmen. Dabei ist zu beachten, dass der Schwerpunkt chinesischer Aktivitäten bisher (noch) nicht auf Deutschland lag. Zudem haben sich die bereits erweiterten Kompetenzen der Aufsichtsbehörden in der Praxis niedergeschlagen. Auf eine weitere Verschärfung sowie Abschottung sollte daher verzichtet werden. Stattdessen sollten Deutschland und die Europäische Union ihre wirtschaftliche Bedeutung für die Volksrepublik dazu nutzen, ein Level-Playing-Field in den Beziehungen mit China zu etablieren.

17 C. Rusche: Chinesische Investoren in Deutschland ..., a. a. O.

18 C. Rusche: Chinesische Beteiligungen und Übernahmen ..., a. a. O.

19 Eigene Berechnung auf Basis PricewaterhouseCoopers: Destination Deutschland, M\&A-Aktivitäten ausländischer Investoren 2019.

20 4,6 Mrd. Euro entfielen 2016 auf die Kuka-Übernahme und 5,8 Mrd. Euro 2017 auf die ista-Übernahme. 